\title{
An Energy-Efficient and Routing Approach for Position Estimation using Kalman Filter Techniques in Mobile WSNs
}

\author{
Y. Donoso, G. A. Montoya, F. Solano
}

\author{
Yezid Donoso*, Germán A. Montoya \\ System and Computing Engineering Department \\ Universidad de Los Andes \\ Bogotá, Colombia \\ ydonoso@uniandes.edu.co,ga.montoya44@uniandes.edu.co \\ *Corresponding author: ydonoso@uniandes.edu.co
}

\section{Fernando Solano}

Warsaw University of Technology

Warsaw, Poland

fs@tele.pw.edu.pl

\begin{abstract}
Mobile Wireless Sensor Networks is being an attractive field due to its applicability to an increasingly amount of mobile scenarios such as wild monitoring, disaster prevention, object guidance and health monitoring. In addition, since the sensors have limited batteries, data routing has to be planned strategically in order to extend the battery lifetime as much as possible [1] [2].

In this paper, we assume GPS free sensor devices, where considering a predictive technique to estimate the sensor position in a circular trajectory scenario can be useful to know when the sensor will be as close as possible to a sink, and then, help us to reduce the energy consumption by the fact of transmitting data at a short distance respect to the sink. In this paper, we propose an predictive algorithm based on Kalman filter techniques to estimate the proper time at which the sensor is close as much as possible to a sink, in order to reduce the energy consumption in the sensor. Specifically, we propose the usage of two Kalman Filters. One Kalman Filter is used for estimating the Received Signal Strength Indicator (RSSI) level based on several control packets received at the sensor device. This RSSI estimation indicates the distance from the mobile sensor device to the sink at a given time. The second Kalman Filter, based on the outputs from the first Kalman Filter, estimates the angular velocity and the angle of the mobile sensor device at a given time. Once this information is processed, it is possible to estimate the mobile sensor position in a circular trajectory in order to determine how much close is the mobile sensor device respect to the sink. In addition, the communication channel noise may affect the packet content, generating non-accurate information measurements at the receptor. For this reason, our proposal is evaluated under different noise channel levels and compared against a traditional technique. Our predictive routing algorithm shows better results in terms of distance accuracy to the sink and energy consumption in noisy communication channels.
\end{abstract}

Keywords: MWSN, position estimator, Double Kalman filter.

\section{Introduction}

The advances of Wireless Sensor Networks (WSN) have allowed attaching the sensors to an entity such as an object, animal or human, to monitor a physical variable presented in its environment. However, the sensors are equipped with limited batteries whereby it is required to implement energy efficient routing techniques to extend the lifetime of the sensors. In addition, the sensors mobility can be disadvantageous for those ones that are distant from a sink, since 
this indicates employing more power transmission to set a communication link, which represents more energy consumption [1] [2].

Given the scenario described above, a possible solution would consist to implement energy efficient routing techniques considering the sensor position to know accurately when the sensor is near to a sink. Some of these solutions propose the usage of sensors equipped with GPS devices, called GPS non-free approaches. However, these GPS non-free solutions have in most of cases drawbacks such as high implementation costs, delays for acquiring position information and non-accurate position information [3]. In addition, these types of solutions require an extra chip for the GPS [4], whereby more energy consumption is experimented.

In this sense, GPS free solutions can be a viable option to solve the problem described above through using predictive techniques to estimate when it is pertinent to send data packets a sink [3]. However, the prediction processes must take into account external factors such as the inherent noise introduced by the wireless channel in the environment. For this purpose, one of the most well-known and often-used tool is the Kalman Filter. It is basically a predictorcorrector type estimator that minimizes the estimated error covariance when some presumed conditions are accomplished. The Kalman Filter has been the subject of extensive research and application, for instance in the area of autonomous or assisted navigation [8]. Thanks to the advances of digital computing, currently the Filter is practical in many applications, and due to its simplicity and robustness it can be used in sensor devices since they have severe computation and memory constraints [2], [8].

In this paper, we propose an energy efficient routing algorithm based on Kalman Filtering techniques to predict a future state of the system in order to determine the accurate time to send data packets from a sensor to a sink in despite of the wireless channel noise. Specifically, we propose the usage of two Kalman Filters. The first Kalman Filter is employed for estimating the RSSI level based on several control packets received at the sensor device. This RSSI estimation indicates the distance from the mobile sensor device to the sink at a given time. The second Kalman Filter, based on the outputs from the first Kalman Filter, estimates the angular velocity and the angle of the mobile sensor device at a given time. Once this information is processed, it is possible to estimate the mobile sensor position in a circular trajectory in order to determine how much close is the mobile sensor device respect to the sink.

The remainder of the paper is organized as follows: Section II shows in detail the problem description as well as the theoretical basis and the algorithm of our solution is shown in section III. In Section IV, we present results for the predictive approach and the classical solution showing the advantages and disadvantages of them. Finally, in Section V, we show conclusions and present future directions of our work.

\section{Problem Description}

The scenario to be analysed is shown in the figure 1, which it is compound of at least two sinks, $s_{1}$ and $s_{2}$, and one sensor (target) $t$ attached to an entity (human, animal or object) describing a circular trajectory. For illustrative reasons, our application case will be an athlete monitoring scenario, in which it is assumed a sensor will be attached to an athlete for sending and collection of physiological data. The scenario characteristics is described in detail below.

In the circular trajectory, the target will have an angular velocity $w_{k}$ which it is assumed to be constant. However, the angular velocity and the circular trajectory are assumed no ideal such as in real life application scenarios, whereby we assume these variations follow a Gaussian distribution with mean $\mu=0$ and with a standard deviation $\sigma$ defined in detail later. This means the target angular velocity will increase or decrease according to a Gaussian distribution along the circular trajectory, and likewise, the radius $r$ of the circular trajectory will have a little 


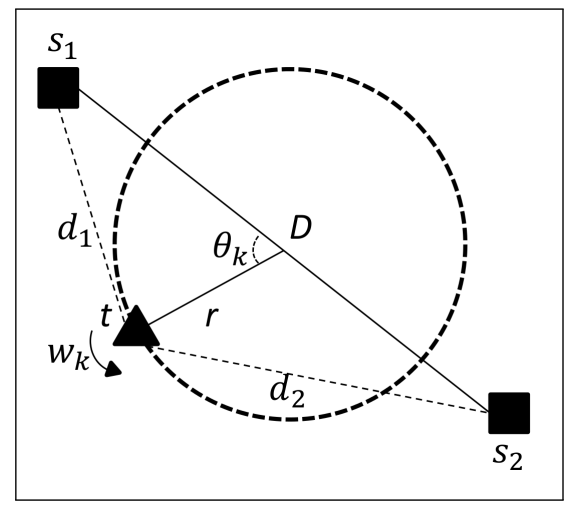

Figure 1: Application scenario.

variation in time following also a Gaussian distribution. In addition, it is assumed there are two sinks located close to the road, $\left(s_{1}\right.$ and $\left.s_{2}\right)$ with a fixed $D$ distance between them. Further, we assume there is always a noisy wireless link communication between sinks and the target $t$.

Due to the target is compound of a wireless sensor with a limited battery, it must be used efficiently in order to extend its lifetime for future usage. The target has to collect relevant information which must be sent to any sink each certain time in order to use efficiently the sensor buffer, which also is limited. For our case, we assume the best time to send data is when the target is as close as possible to a sink. Due to, for our case, it is not possible to know at any time the accurate position of the target, knowing the distance between the target and a sink could be a encouraging start to determine the target position. The distance can be inferred by extracting the RSSI value of a packet, however, the noise can disturb our measurements, and then, affecting our position estimation. As a result, our problem consists how to send the data collected by the target in an efficient energy way without knowledge of the target position in the circular trajectory, taking into account the negative noise effects caused by the wireless channel to estimate distances.

Given the problem above, a classical/intuitive solution, proposed by us, would be the target receiving a packet at each time with its correspondent RSSI value to establish how close it is from a sink. In a similar way, our novel proposal also uses the RSSI attribute, but more times than the classical solution, employing an algorithm which use the Kalman filter basis.

\section{Proposed solution}

Taking into account the figure 1, our proposed solution would estimate when the target is close to a sink based on $d_{1}, d_{2}$ and $D$ distances, the angular velocity $W_{k}$ and the angle variation $\theta_{k}$. Once these variables are calculated, it is possible to estimate when the target will be as close as possible to a sink.

The $d_{1}$ and $d_{2}$ distances can be inferred through the RSSI measured when the sinks and the target interchange control messages. However, a RSSI value can be unstable since it is affected by the wireless channel, in other words, by the noise introduced by the channel itself. In this sense, it could be not enough to estimate the distance based only on a unique RSSI value, because it could be a wrong estimation. For this reason, in order to do a suitable estimation of distances, it is required to collect more than one RSSI values, which, in turn, will provide proper estimated values of $w_{k}$ and $\theta_{k}$ variables. The quality of these variables is very important since it will allow to estimate properly the moment in which the target should send its data to a sink. As a result, 
the more RSSI values are sampled, the higher quality of $w_{k}$ and $\theta_{k}$ is obtained. However, the above will produce more energy consumption because more control packets are transmitted, and then, it has to be considered at setting parameters for the simulation.

In addition, it is necessary to determine how many control packets should be sent in order to obtain suitable distance estimations and, at the same time, saving energy consumption to extend the lifetime of the sensor, and avoiding the noise negative effects.

In terms of energy consumption, it is necessary to consider consumption by transmission and reception in the sensor. For transmission, the energy consumption is defined as $\left(E_{\text {elec }}+\right.$ $\left.E_{\text {amp }}\right) * k * D^{n}$, while for reception, it corresponds to $E_{\text {elec }} * k$. $E_{\text {elec }}$ is the energy consumption for codification, modulation and filtering, and $E_{a m p}$ corresponds to energy consumption for the TransmitterPower Amplifier [5]. Additionally, the following parameters values are assumed: $E_{\text {elec }}$ is $50 \mathrm{~nJ} / \mathrm{bit}, E_{a m p}$ is $100 \mathrm{pJ} / \mathrm{bit} / \mathrm{m}^{2}, n$ is assumed to be 2 and $D$ corresponds to the distance to send a $k$-bit packet [6].

In addition to determine the number of packets for distance estimations, it is necessary to define a $\Delta t$ at which the samples will be taken. If $\Delta t$ is too high, that is, the frequency to take samples is too low, is highly probable that the closest distance to a sink generated by the solution will be too high respect to the theoretical best distance to a sink. On the contrary, if $\Delta t$ is too low, the solution will be quite better than the previous, but at the expense of a lot of energy consumption.

\subsection{Kalman filter applied to our proposal}

The Kalman filter is a recursive algorithm, which is compound of two phases, as shown in the figure 2, that are performed iteratively: a Prediction phase and a Correction phase. For space reasons, the Kalman filter basis are omitted, but if you are interested in the basis details, you can refer to [7] [8].

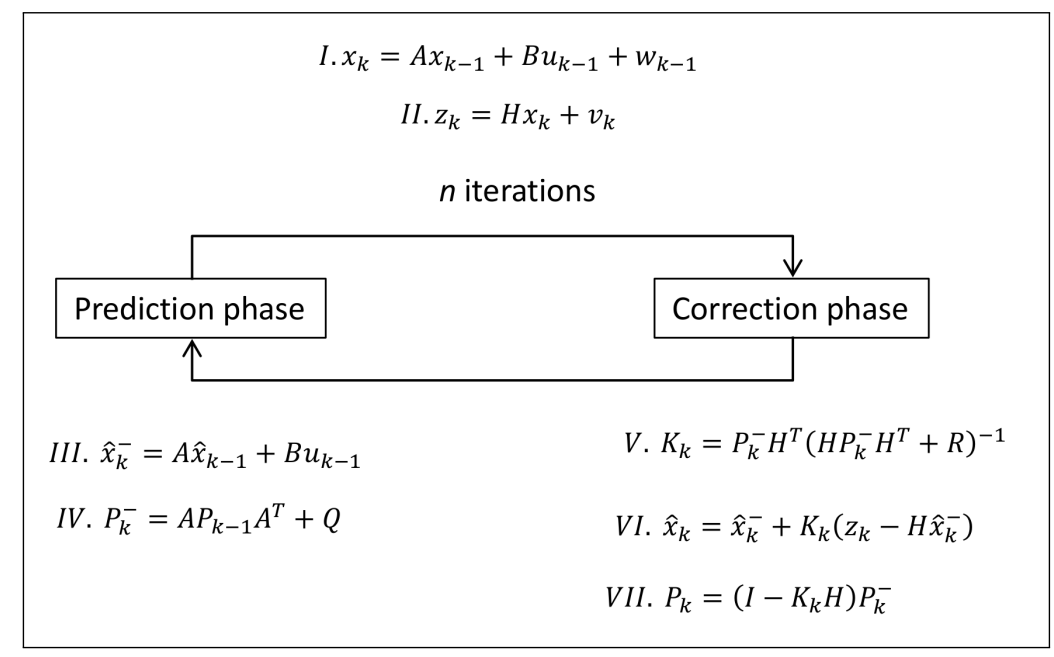

Figure 2: Kalman filter algorithm phases.

We propose to perform two Kalman Filters as it is shown in the Figure 3. One Kalman filter for RSSI and distances $\left(d_{1}\right.$ and $\left.d_{2}\right)$, and the other one for $\theta_{k}$ and $w_{k}$ estimations. For the first one, since we are assuming the RSSI and distances estimated should converge to a constant value, the matrix $A$, indicated in Figure 2 expressions (I), (III) and (IV), must be equal to "1" according to the Kalman Filter theoretical basis. In addition, the observation matrix $H$ also has 


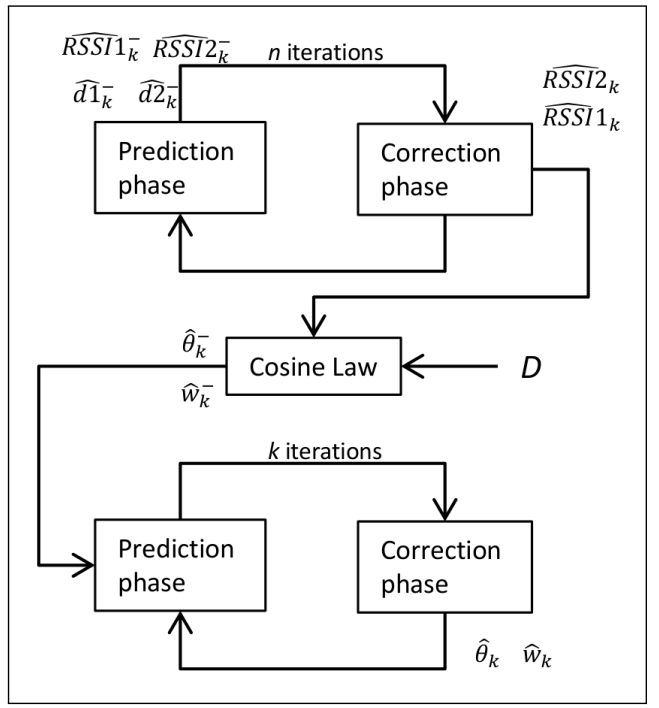

Figure 3: Kalman filter block diagram implementation.

a "1" value, because only one variable is observed. For the second Kalman filter, the expression (I) in the Figure 2 can be defined as follows, where $A$ matrix can be inferred:

$$
x_{k}=\left[\begin{array}{l}
\theta_{k} \\
w_{k}
\end{array}\right]=\left[\begin{array}{ll}
1 & \Delta t \\
0 & 1
\end{array}\right]\left[\begin{array}{l}
\theta_{k-1} \\
w_{k-1}
\end{array}\right]+w_{k-1}
$$

In the expression above, notice that the angular velocity is indicated in uppercase and the process noise in lowercase. The $A$ matrix expressed in (1) indicates the transition model state which, basically, defines the circular trajectory of the target. Notice that $\Delta t$ indicates the time in which we want generate the next $k^{\text {th }}$ state of the system. The $\Delta t$ should not be selected arbitrary since a too high value of $\Delta t$ will generate excellent results but at the expense of a high energy consumption, and a too low value of $\Delta t$ will not afford accurate target position.

In the Algorithm 1 is presented the main aspects of our solution. In addition, a block diagram in the figure 3 is shown to complement the pseudocode explanation.

In line 2 , the target is moved with an angular velocity $w_{s}$, an angle displacement $\theta_{s}$ during a lapse of $\Delta t_{s}$ for the movement simulation of the sensor, which is indicated with the index $s$. In line $3, \Delta t_{k}$ indicates the lapse in which the samples for Kalman algorithm will be taken. In 6, $\mathrm{Paq}_{t x}$ indicates the number of packets with RSSI values in order to do a proper estimation using a Kalman algorithm, that is, to generate the values indicated in line 8 and 9 . In 10, it is predicted the RSSI for $s_{1}$ and $s_{2}$ according to the previous corrected values using a Kalman filter. In 11 , the estimated distances to $s_{1}$ and $s_{2}$ are predicted based on the estimated RSSI previously obtained.

In 13 , a Kalman filter is applied over $\hat{d} 1_{k}^{-}$and $\hat{d} 2_{k}^{-}$to obtain the corrected values $\hat{d} 1_{k}$ and $\hat{d} 2_{k}$. Once the distances has been calculated in 13, in line 14 the cosine law is applied to calculate $\bar{\theta}_{k}$ based on the values of $\hat{d} 1_{k}, \hat{d} 2_{k}, r$ and $D$ (Figure 1 ). Due to $\overline{\theta_{k}}$ and $\Delta t_{k}$ is possible to obtain $\bar{W}_{k}$ based on the expression (1). Notice that $\overline{\theta_{k}}$ and $\Delta t_{k}$ correspond to the observed values $z_{k}$ indicated in the expression (II) of the Figure 2. In line 15 and 16, based mainly on observed values $\overline{\theta_{k}}$ and $\bar{W}_{k}$; the transition model state matrix $A$ described in the expression (1); and through the Kalman filter theory is possible to predict and correct the values of $\theta$ and $W$, that is, $\hat{\theta}_{k}^{-}$and $\hat{W}_{k}^{-}$, and $\hat{\theta}_{k}$ and $\hat{W}_{k}$ respectively. Once these values are calculated the $(x, y)$ coordinates can be found and determine how close is the target from the sinks. 


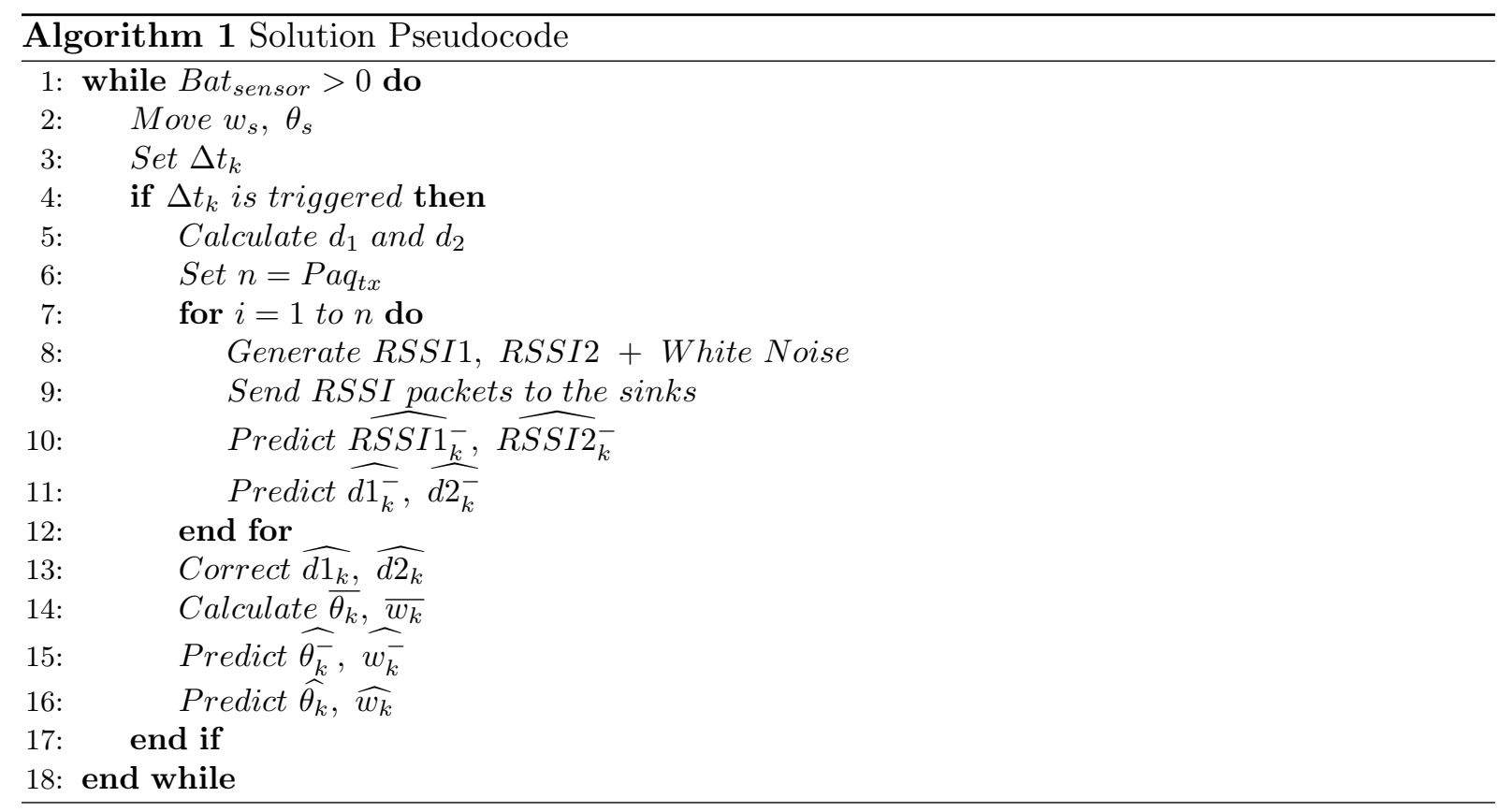

\section{Results}

Before to show the results, it is necessary to present the following figures, which represent some performance aspects of the traditional and our proposed solution.
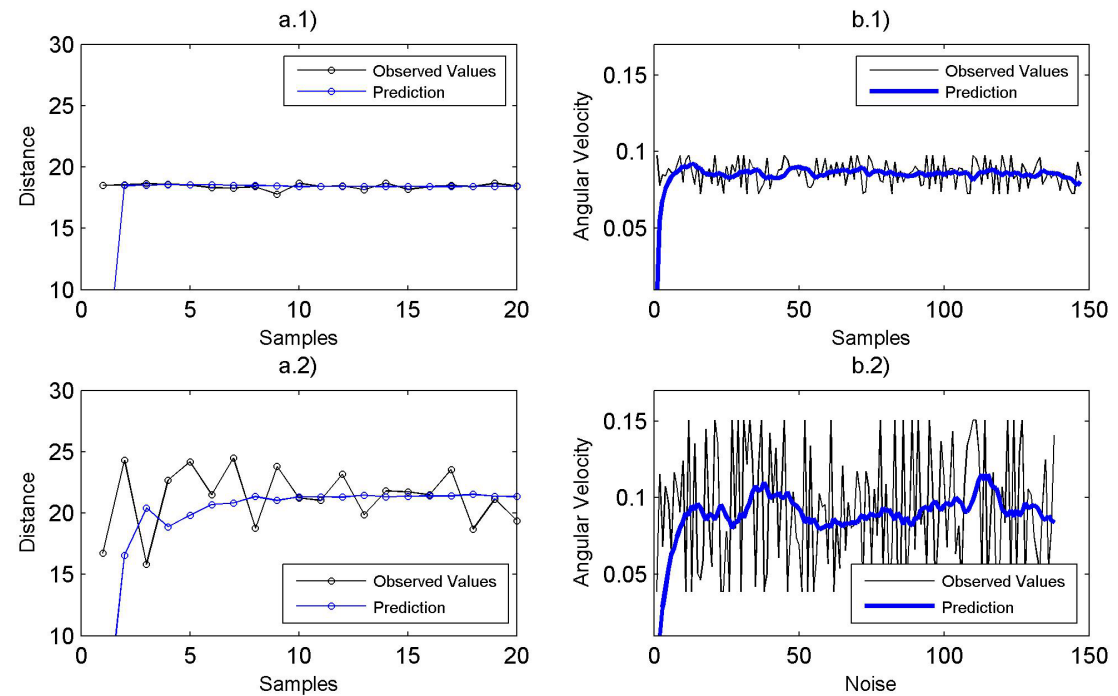

Figure 4: Performance results for traditional and our proposed solution.

The figures a.1) and a.2) show the prediction based on Kalman for a noise of 0.01 and 0.1, respectively, and for 20 packets to determine a distance at a given time. In the figure a.1), as the noise is lower than a.2), the prediction is more accurate than a.2). In the figures b.1) and b.2) is presented the delta angular velocity for a noise of 0.01 and 0.1 , respectively, in order to determine the target position in a given time. In b.2), as the noise is quite high, the prediction is more variable than b.1). As a result, in b.1) the target position is more accurate than b.2). 
For c) and d) figures, the main simulation parameters were: $\Delta t_{k}=700 \mathrm{~ms}, P a q_{t x}=200$, DataSizeforTransmission equal to $500 \mathrm{~KB}$, Perimeter $=400 \mathrm{~m}$ and TargetSpeed $=5.52 \mathrm{~m} / \mathrm{s}$. In c) it is presented the energy consumption for both traditional and our proposed solution according to different noise values. For $0.1,0.2$, and 0.3 noise values, the traditional has a lesser energy consumption than our proposed solution. However, for values higher than 0.3, our proposed solution presents a lesser energy consumption because the traditional solution behaviour is affected by the high noise level. The performance is affected due to sending extra data transmission caused by the high noise present in the communication channel. In d), the distance from the target and the sink 2 is calculated at the moment of data transmission for both traditional and our proposed solution. From 0.1 to 0.3 the performance for both are quite similar. However, for values higher than 0.3 our algorithm outperforms classic solution since it less prone to disturbances caused by noisy wireless channels.

\section{Conclusions}

In this paper, we propose a predictive algorithm based on Kalman filter techniques to estimate the proper time at which the sensor is close as much as possible to a sink, in order to reduce the energy consumption in the sensor.

We propose an energy efficient routing algorithm based on Kalman Filtering techniques to predict a future state of the system in order to determine the accurate time to send data information from a sensor to a sink. The theoretical basis and operation of our algorithm is explained in detail, and its performance is evaluated for an athlete monitoring scenario for illustrative reasons.

The main conclusion of our proposed solution is about its performance in noisy communication channels. It means that the energy consumption of our algorithm is lesser than the traditional method, presenting less impact for noisy communication channels.

\section{Acknowledgement}

This research paper was made possible through the help and support from the European Union Project GOLDFISH at Framework Programme 7.

\section{Bibliography}

[1] I. F. Akyildiz and M. C. Vuran (2010), Wireless Sensor Networks, John Wiley \& Sons.

[2] J. Zheng and A. Jamalipour (2009); Wireless Sensor Networks: A Networking Perspective, Wiley-IEEE Press, ISBN: 978-0-470-16763-2.

[3] D. Gavalas, C. Konstantopoulos, B. Mamalis and G. Pantziou (2010); Mobility Prediction in Mobile Ad Hoc Networks, Book chapter for Next Generation Mobile Networks and Ubiquitous Computing by S. Pierre, IGI Global, 226-240.

[4] B. Buchli, F. Sutton and J. Beutel (2012); GPS-Equipped Wireless Sensor Network Node for High-Accuracy Positioning Applications, Wireless Sensor Networks Lecture Notes in Computer Science, Springer, 7158: 179-195.

[5] S. Li, X. Ma, X. Wang, M. Tan (2011); Energy-efficient multipath routing in wireless sensor network considering wireless interference, Journal of Control Theory and Applications, 9(1): 127-132. 
[6] P. S. Boluk, S. Baydere, A. E. Harmanci (2011); Robust Image Transmission Over Wireless Sensor Networks, Mobile Networks and Applications, 16(2): 149-170.

[7] P. S. Maybeck (1979); Stochastic Models, Estimation and Control, Mathematics in Science and Engineering, Vol.141.

[8] G. Welch and G. Bishop (1995); An Introduction to the Kalman Filter. Technical Report. University of North Carolina at Chapel Hill, Chapel Hill, NC, USA. 